Sessions d'étude - Société canadienne d'histoire de l'Église catholique

\title{
L’apport de l'Église mascoutaine à celle de Sherbrooke
}

\section{Jean Mercier}

Volume 33, 1966

URI : https://id.erudit.org/iderudit/1007318ar

DOI : https://doi.org/10.7202/1007318ar

Aller au sommaire du numéro

Éditeur(s)

Les Éditions Historia Ecclesiæ Catholicæ Canadensis Inc.

ISSN

0318-6172 (imprimé)

1927-7067 (numérique)

Découvrir la revue

Citer cet article

Mercier, J. (1966). L’apport de l'Église mascoutaine à celle de Sherbrooke.

Sessions d'étude - Société canadienne d'histoire de l'Église catholique, 33, 25-30.

https://doi.org/10.7202/1007318ar

Tous droits réservés @ Les Éditions Historia Ecclesiæ Catholicæ Canadensis Inc., 1967
Ce document est protégé par la loi sur le droit d'auteur. L’utilisation des services d'Érudit (y compris la reproduction) est assujettie à sa politique d'utilisation que vous pouvez consulter en ligne.

https://apropos.erudit.org/fr/usagers/politique-dutilisation/ 


\section{L'apport de l'Église mascoutaine à celle de Sherbrooke}

L'année 1966 marque le 15e anniversaire de la fondation de l'archidiocèse de Sherbrooke. Le 2 mars 1951, Pie XII érigeait, par la bulle apostolique Universi dominici gregia, la métropole ecclésiastique de Sherbrooke, lui donnait deux suffragants, les diocèses de Nicolet et de Saint-Hyacinthe, proclamait le nom de son premier titulaire, Mgr Philippe Desranleau. Cette décision romaine que Mgr l'Evêque de Sherbrooke attendait impatiemment depuis nombre d'années, provoqua chez lui une véritable explosion de joie dont les éclats apparaissent dans ses circulaires. Voici quelques extraits d'un discours qu'il a prononcé devant Mgr Antoniutti :

" L'Eglise de l'Estrie ${ }^{1}$ jubile à son tour ... elle chante très haut sa joie et sa reconnaissance. Elle ne peut s'empêcher de célébrer l'œuvre de la Providence qui sait, avec la lenteur de la sagesse et la sécurité de la prudence, arriver à ses fins...

* Chaque expression sera en dessous de l'intensité de notre joie : si je vous dis que Sherbrooke est la tête et le cœur de l'Estrie ... je ne vous apprends rien de nouveau... Oui, Sherbrooke méritait de devenir la métropole de l'Estrie, parce qu'elle est la ville de la beauté parfaite, le joyau de toute la terre canadienne ${ }^{2}$.

Dans une correspondance avec Mgr Arthur Douville, le nouvel Archevêque élu écrit le 7 mars 1951 :

* Le Saint-Siège a érigé la province ecclésiastique de Sherbrooke et lui a donné Saint-Hyacinthe et Nicolet comme suffragants. Il a ainsi répondu à un vœu déjà exprimé en 1910 par toute la province de Montréal. Sherbrooke a été choisi, j'en suis heureux, mais croyez bien que si Saint-Hyacinthe ou Trois-Rivières avaient été préférés, ils auraient eu mon assentiment. "(Archives de l'archevêché de Sherbrooke.)

Trois quarts de siècle auparavant, plus exactement le 28 août 1874, Pie X taillait le diocèse de Sherbrooke dans ceux de Québec, des TroisRivières et de Saint-Hyacinthe. Ce dernier fit cadeau à Sherbrooke des paroisses et missions suivantes éparpillées dans 18 cantons, mais situées sur la rive nord de la Saint-François : Sainte-Praxède de Brompton,

1 L'Estrie est l'autre nom régional donné aux Cantons de l'Est. Voici un extrait du rapport de la séance de linguistique tenue le 5 mars 1951 : « Académie canadienne-française. Le Comité de linguistique accepte le terme «Estrie 》 pour désigner les "Cantons de l'Est 》 et en recommande l'usage. Pour copie, le secrétaire perpétuel Guy Frégault. 》

L'Estrie, Jean Mercier, Editions Paulines, Sherbrooke, 1964, p. 127.

2 Lettre pastorale, vol. XVI, $\mathrm{n}^{\circ} 72$, p. 174, 176, 177. (Mandements diocésains de Sherbrooke.) 
Saint-Thomas d'Aquin de Compton, Saint-Edmond de Coaticook, SainteHedwige de Clifton, Saint-Herménégilde de Barford, Saint-Venant de Hereford, Saint-Camille de Cookshire, Sacré-Cœur de Stanstead, SainteCatherine de Hatley, Saint-Patrice de Magog, Saint-André de Sutton, Sainte-Anne de Stukeley, Notre-Dame de Bonsecours, Valcourt, SaintEtienne de Bolton, les missions de Saint-Roch d'Orford et de SaintWilfrid de Barnston.

La communauté mascoutaine a été pour Sherbrooke une mère généreuse et une fille dévouée. Sans compter la centaine de prètres qu'elle a fournis à notre ministère, elle nous a donné quatre de nos six chefs spirituels diocésains, a collaboré avec l'équipe du départ, nous a envoyé un contingent de pasteurs valeureux, dont les frères Adam ${ }^{3}$, de religieuses dépareillées, de laïques de premier plan.

Saint-Hyacinthe nous a mis au monde et il continue à nous développer, à nous aiguillonner vers les progrès par son exemple et par sa coopération. La gratitude quasi centenaire qui logeait dans nos cœurs, nous aimerions l'exprimer à l'occasion de ce congrès.

Le diocèse de Saint-Hyacinthe est le frère jumeau, non identique, de celui de Trois-Rivières; les deux naissent le 8 juin 1852. D'abord, suffragant de Québec pendant 34 ans (1852-1886), Saint-Hyacinthe dépendit de Montréal au cours de 65 années (1886-1951) avant d'être soudé à Sherbrooke.

Lorsqu'en août 1874 Pie IX créa notre diocèse, les curés de la côte nord de la rivière Saint-François acceptèrent, non sans un pincement de cour, de passer d'un diocèse opulent et stable à un autre à peine ébauché, en pleine période de colonisation, mais promis à un brillant avenir. Nous voudrions rendre un vibrant hommage à tous ces pionniers mascoutains de la pastorale en marche, à ces bâtisseurs de notre chrétienté diocésaine, en nous arrêtant un moment devant la personnalité de l'abbé Elie Dufresne, que l'histoire nomme le grand vicaire Dufresne. Ce prêtre a été le véritable précurseur de Mgr Antoine Racine, le premier évêque de Sherbrooke; il lui a préparé la voie et a jeté les bases des cuvres paroissiales, scolaires et hospitalières de notre région de l'Estrie. La société d'histoire régionale et le comité du centenaire de Sherbrooke ont fait couler dans le bronze le texte suivant honorant sa mémoire :

- A la perpétuelle mémoire du Grand vicaire Alfred-Elie Dufresne, apôtre de la paix et de la charité. Il fonda puis desservit seul, pour un temps, les missions de Magog, Hatley, Cookshire, Stoke, Brompton, Lennoxville, Orford, et, durant toute sa vie curiale à Sherbrooke (18531891), fit prospérer le catholicisme au milieu des races et des croyances diverses. On admire ici l'un des monuments dus à son amour intelligent pour la science et la religion. *

3 Bastion sacerdotal en Estrie, Jean Mercier, Editions Paulines, Sherbrooke, 1965, p. 101 à 106. 
Cette plaque commémorative, fixée sur le flanc du Mont NotreDame ${ }^{4}$, rappelle aux visiteurs les faits et gestes de ce missionnairecolonisateur. En vous référant aux lettres de Mgr J.-Charles Prince écrites au curé Dufresne, nous apprenons que la nouvelle chapelle Saint-Colomban de Sherbrooke, ouverte au culte depuis 1827, fut bénite par l'évêque mascoutain, Mgr Prince, le 14 janvier 1855. Le 7 mars 1872, l'Ordinaire de Saint-Hyacinthe substitua, devant la montée grandissante de l'élément canadien-français, à celui du saint irlandais, le nom de Saint-Michel.

On détecte l'influence du curé Dufresne dans l'organisation des premières écoles sherbrookoises, dans le lancement de l'Institut littéraire de Sherbrooke (futur collège industriel qui deviendra le séminaire Saint-Charles-Borromée), dans l'achat des terrains pour l'hospice du Sacré-Cour, dans la mise en marche de la colonisation avec ce grand patriote mascoutain, J.-A. Chicoyne, agent des terres dans nos cantons depuis 1847. Grâce à cette société de colonisation animée par M. Chicoyne, les Mascoutains affluèrent nombreux dans nos townships.

Sherbrooke doit encore à Saint-Hyacinthe quatre de ses six chefs diocésains : NN.SS. Paul LaRocque, évêque de 1893 à 1926, Hubert Chalifoux, auxiliaire de 1914 à 1922, Philippe Desranleau, coadjuteur de 1937 à 1941, évêque titulaire de 1941 à 1951 et archevêque de 1951 à 1952 et Georges Cabana, notre archevêque actuel depuis 14 ans $^{5}$.

Les relations entre les diocèses de Sherbrooke et de Saint-Hyacinthe ont toujours été empreintes de cordialité et marquées au coin de la collaboration.

Mgr Paul LaRocque, le pasteur princier et le successeur de Mgr Antoine Racine, l'évêque bâtisseur, est né au Monnoir. Curé de la paroisse-mère en la cité mascoutaine, il apprit lors d'un voyage aux Etats-Unis, sa promotion comme évêque de Sherbrooke.

Le chanoine Paul LaRocque, accompagné de son frère, l'abbé Charles, visitait une grande cité américaine. L'abbé Charles raconte : - Nous étions à Chicago. C'était le 26 septembre 1893. Nous avions passé la plus grande partie de la journée dans la bâtisse des machines, et vers les quatres heures, p.m., nous étions à admirer le fonctionnement de la grande presse du Chicago Daily News. Or, c'est précisément sur ce journal, édition du mardi soir que s'imprimaient sous nos yeux les quelques lignes suivantes : « The Pope appoints Bishops. The Pope has c appointed the Rev. Father Edward Dunn of the Chicago diocese, bishop - of Dallas, Texas. The Rev. Father Paul LaRocque has been appointed - bishop of Sherbrooke, Canada. .

4 Institution fondée en 1857 et dirigée par les søurs de la Congrégation de Notre-Dame, sise à 114, rue de la Cathédrale, Sherbrooke.

S On trouvera, dans le livre suivant, des détails biographiques sur ces différents personnages : Autour de Mena 'Sen, Jean Mercier, Editions Paulines, Sherbrooke, 1964. 
Mgr Hubert Chalifoux appartenait à une famille distinguée de Saint-Hyacinthe. Il est né le 2 juin 1850. Ordonné prêtre, ce pasteur se signala surtout comme un intendant fidèle et intègre. Administrateur du diocèse de Sherbrooke à partir de 1891, il assuma aussi, l'année suivante, la fonction de vicaire général.

Nos deux archevêques, Mgr Philippe Desranleau, natif de SaintSébastien, et Mgr Georges Cabana, natif de Granby servirent à différents postes dans le diocèse de Saint-Hyacinthe avant d'accéder à la charge de métropolitains de Sherbrooke. 'lous deux sont des gloires prestigieuses de l'Eglise mascoutaine.

Notre Eglise diocésaine doit beaucoup aux communautés religieuses d'origine ou de rayonnement mascoutains : les sœurs de la Présentation de Marie (1870), de la Charité de Saint-Hyacinthe (1875), du PrécieuxSang (1895) et de Saint-Joseph (1949).

C'est à M. l'abbé Jean-Baptiste Chartier que l'on doit l'arrivée des sœurs de la Présentation de Marie dans le diocèse. Appelé en 1863 à desservir la mission de Saint-Edmond de Coaticook, il en devint premier curé en 1868, assisté de l'abbé Amédée Dufresne. Mgr Charles LaRocque accorda au curé la permission de fonder un couvent à Coaticook et l'engagea à s'adresser d'abord à la Congrégation Notre-Dame de Montréal, persuadé que cet institut pouvait faire les frais de la construction. Des circonstances particulières empêchant les filles de Mère Bourgeoys de se charger de cette œuvre, Messire Chartier obtint de Mgr LouisZéphirin Moreau les services des Sœurs de la Présentation. Pour décrire l'atmosphère de l'époque et des lieux où essaimèrent les valeureuses mascoutaines, nous livrons quelques extraits des chroniques du couvent :

* La population protestante voyait d'un mauvais œil s'établir une institution religieuse, et quelques fanatiques s'efforcaient de raviver les préjugés. Ces dispositions impressionnaient les enfants protestants. Le 17 novembre 1870, deux mois après l'arrivée des Religieuses, comme elles revenaient d'une promenade avec leurs élèves, quelques enfants de l'école protestante, croyant que les Religieuses étaient des personnes que l'on pouvait insulter impunément, voulurent s'amuser en leur jetant des boules de neige, qu'ils accompagnaient de leurs éclats de rire. Les Religieuses forcèrent leurs élèves indignées à garder le silence et ellesmêmes conservèrent une attitude calme et paisible. Les parents confus châtièrent leurs enfants, et le ministre anglican, le Rev. John Foster, fit signer à ces derniers une lettre d'excuses que conservent les « Annales du Couvent s. Il écrivit lui-même à M. le Curé, le priant de faire agréer aux Religieuses ses regrets personnels ${ }^{6}$.

Coaticook, qu'on se plaît à appeler \& La Perle de l'Estrie », a été le berceau de la Présentation de Marie. Actuellement 173 Religieuses de la Présentation de Marie œuvrent dans 19 maisons diocésaines : membres

- Annales du Couvent, p. 5 à 9. 
de l'administration, enseignantes, infirmières, malades, sœurs à la retraite, convalescentes, préposées à la maison Rivier (maison de retraites fermées). Elles s'y dévouent par l'action, la prière ou la souffrance.

Une compilation récente des statistiques faites dans les maisons « présentines » révèle que 410 jeunes filles optèrent pour la vocation religieuse, et de ce nombre, 182 se dirigèrent vers le noviciat de la Présentation de Marie.

Le 21 avril 1875, à la demande de Mgr Antoine Racine, secondé par l'abbé Dufresne, son vicaire général, quatre sœurs Grises de la Charité de l'Hôtel-Dieu de Saint-Hyacinthe, essaimèrent à Sherbrooke pour y instaurer le travail d'hospitalisation.

Les vaillantes pionnières de l'hospice du Sacré-Cœur, mettant au service de la charité, leurs vies, leurs travaux et leurs dévouements, généreusement aidées par le clergé et la population, venaient ici, prendre soin des vieillards indigents, recueillir les orphelins et procurer soins et soulagement aux malades. Pendant les vingt premières années, ces trois œuvres ont été développées, après quoi un nouvel essor fut donné à l'œuvre des malades.

Le petit hospice de la rue Belvédère, modeste habitation, devenu insuffisant, fut laissé pour l'édifice actuel dans le vent de la côte King est. Le développement scientifique et technique du soin des malades, nécessita dans la suite, sous la vigilance de Mgr Paul LaRocque, le déplacement de l'œuvre dans un hôpital particulier, l'hôpital général SaintVincent-de-Paul, qui vit bientôi l'ouverture d'une école d'infirmières. Plus tard, l'hôpital d'Youville destiné aux maladies chroniques s'ajoutera à l'hospice du Sacré-Cour. C'est le missionnaire mascoutain Elie Dufresne qui bénit avec joie l'hôpital général de Sherbrooke.

Trois œuvres temporaires locales ont aussi bénéficié de la sollicitude des Sœurs de la Charité, celle de la garderie Sainte-Elisabeth, ainsi que l'entretien de l'hôpital civique (que le peuple avait baptisé hospice de picotés et l'oeuvre de la soupe au temps de la crise économique).

Le grain de sénevé de 1875, s'est développé et est devenu un grand arbre de charité. La phalange de plus d'un demi-millier de Religieuses, filles de la bienheureuse Marguerite d'Youville, qui depuis près d'un siècle, se sont succédé dans l'œuvre hospitalière de Sherbrooke, ont poursuivi leur but, fidèles à leur mission de charité et de dévouement, à elles confiée par l'Eglise de Saint-Hyacinthe et les autorités religieuses de son Hôtel-Dieu.

Hommage à ces Religieuses mascoutaines dignes émules de la bienheureuse Marguerite d'Youville, que Jean XXIII a proclamée * Mère à la charité universelle ».

Nos Adoratrices du Précieux-Sang se disent très reconnaissantes à l'Eglise mascoutaine de leur avoir donné naissance, au diocèse de Sherbrooke, le 14 septembre 1895. 
Cette fondation d'une communauté contemplative, la première du genre en Estrie, était accordée par Mgr Louis-Zéphirin Moreau, à la demande de Mgr Paul LaRocque, lui-même natif du diocèse de SaintHyacinthe comme on le sait.

Cousin de Mgr LaRocque, fondateur conjointement avec mère Catherine-Aurélie-du-Précieux-Sang de l'institut du Précieux-Sang à Saint-Hyacinthe, le 14, septembre 1861, Mgr Paul LaRocque trouvait un généreux collaborateur en la personne de $M$. l'avocat Chicoyne, un ināscoūütäin, puus dssevir maiériellemeni son œuvre, iout près de l'évêché, soit le Parthénon actuel.

Quant à la gouverne spirituelle de la nouvelle communauté, elle fut confiée à un autre personnage éminent de Saint-Hyacinthe, M. l'abbé P.-J.-A. Lefebvre, supérieur du séminaire Saint-Charles-Borromée; 33 années durant, il y exerça son ministère avec un inlassable dévouement.

En plus des fondatrices, mère Marie-Sainte-Ursule et ses sept compagnes venues de Saint-Hyacinthe, le monastère de Sherbrooke aura accueilli six sujets natifs de ce diocèse : et c'est là également, au Noviciat central, que les Religieuses reçoivent leur première formation.

Toutes, à Sherbrooke, gardent un indéfectible souvenir de gratitude aux dévoués évêques et prêtres de l'Eglise mascoutaine au nombre desquels on compte M. l'abbé Xyste Desautels, né à Saint-Hilaire-surRichelieu, chapelain depuis plus de dix ans.

Les sœurs de Saint-Joseph de Saint-Hyacinthe, répondant à la demande des commissions scolaires, des curés comme au désir des parents, acceptèrent de prendre la direction de six écoles dans le diocèse. Assbestos et Bonsecours les accueillent en 1949; puis en 1951 et 1952, c'est au tour des paroissiens de Notre-Dame-des-Bois et de Racine d'ouvrir leurs bras et leurs cœurs à ces éducatrices de choix venues de SaintHyacinthe. Le diocèse de Sherbrooke leur avait donné 47 sujets.

Voilà quelques grands traits de la fresque de l'Eglise sherbrookoise esquissés par les Mascoutains. En regardant les grands artisans de cette œuvre, tous ces bâtisseurs de notre communauté diocésaine, j'évoque spontanément cette pensée du chanoine Lionel Groulx, historien du Canada français, lue dans le Messager des Fondateurs de l'Eglise du Canada : "Il n'est pas indifférent à la vie d'une chrétienté soucieuse de maintenir à bon niveau sa vie spirituelle, de sentir tout près de soi, fils de son sol et fils de son histoire, les héros de sa foi. 》

L'abbé Jean Mercier

Principal de l'Ecole Normale

Notre-Dame du Sacré-Cœur,

Sherbrooke, P.Q. 